\title{
Inflammaging - Altern als Konsequenz chronischer Entzündungen: Das Beispiel Padma 28
}

\author{
Isaac Ginsburg1', Cécile Vennos², Erez Koren³ \\ ${ }^{1}$ Institute for Dental Sciences, Hebrew University-Hadassah Faculty of Dental Medicine, Jerusalem, Israel; ${ }^{2}$ Padma AG, Research Department, \\ Schwerzenbach, Schweiz; ${ }^{3}$ Department of Pharmaceutics, School of Pharmacy, The Hebrew University of Jerusalem, Jerusalem, Israel.
}

m Alter treten spezifische Veränderungen des Immunsystems auf. Dieser Prozess der Immunoseneszenz (Verlangsamung der Immunantworten) wird durch eine Verschiebung des Gleichgewichts zwischen pro- und anti-inflammatorischen Signalwegen hin zu einem proinflammatorischen Immuntyp charakterisiert [1,2]. Als Ursache für die zunehmende Dominanz des zellulären Immunsystems wird eine Erschöpfung des Pools an naiven T-Zellen und ein abnehmendes T-Zell-Repertoire vermutet, bedingt durch die lebenslange Exposition gegenüber endogenen und exogenen proinflammatorischen Faktoren. Im Laufe der Zeit akkumulieren diese und führen zu einer permanenten Aktivierung des angeborenen und/ oder des erworbenen Immunsystems.

Dies hat einerseits eine reduzierte Plastizität der Immunabwehr zur Folge, andererseits führen solche Prozesse zur Entstehung eines proinflammatorischen Milieus im Körper [3,4]. Der daraus entstehende chronische, unterschwellige Entzündungszustand äussert sich in der Zunahme verschiedener Entzündungsfaktoren und wird nach FRANCESCHI mit dem Begriff Inflammaging umschrieben [2,5,6-8]. Inflammaging wird vermutlich verschlimmert durch zusätzliche proinflammatorische Einflüsse wie z.B. persistierenden oxidativen Stress, akute Entzündungen, Übergewicht, Bewegungsmangel oder einen gestörten Schlafrhythmus [9-11].

Die ätiologische Bedeutung chronischer Entzündungsvorgänge bei so genannten Alterserkrankungen und die Erkenntnis, dass verschiedene im Alter häufiger auftretende pathologische Zustände miteinander in Wechselbezie-

Inflammaging bezeichnet einen chronischen Entzündungszustand, der durch altersbedingte Veränderungen des Immunsystems entsteht. Dieser wird als Grundlage vieler chronisch-entzündlicher Alterskrankheiten wie z.B. Arteriosklerose, Diabetes mellitus Typ 2, Morbus Alzheimer und Krebserkrankungen vermutet. Die Entstehung des vorherrschenden proinflammatorischen Milieus und der Verlauf des Entzündungsprozesses wird durch oxidativen Stress beschleunigt und in manchen Fällen verstärkt. Bei Entzündungsprozessen schütten aktivierte Immunzellen ein Arsenal an bioaktiven Stoffen aus, welche synergistisch zusammenwirken und neben positiven Wirkungen auch zu Zell- und Gewebeschäden führen. Im Fall einer manifesten Infektion sind ähnliche Prozesse beschrieben worden. Komplexe Phytotherapeutika (z.B. Padma 28) sind als pleiotrop wirkende Gemische geeignet, diesen krankmachenden Prozess an unterschiedlichen Wirkorten zu unterbinden. So sind etwa neben dem klinischen Effekt von Padma 28 bei Arteriosklerose auch verschiedene antiatherogene Wirkungsmechanismen des Präparats gut dokumentiert. Die Resultate stützen die Hypothese eines "multitarget"-Behandlungsansatzes von chronisch-entzündlichen Erkrankungen mit pflanzlichen Vielstoffgemischen, die hier einen wertvollen Betrag für neue Präventions- und Therapieverfahren liefern können. Sie sind somit geeignet, den Formenkreis von Inflammaging günstig zu beeinflussen, die Entstehung von Folgeerkrankheiten zu verlangsamen bzw. in manchen Fällen zu verhindern.

Schlüsselwörter: Inflammaging, Immunoseneszenz, chronische Entzündung, geriatrische Erkrankungen, Padma 28

\section{Inflammaging - Aging as a Consequence of Chronic Inflammation: The Example of Padma 28}

Inflammaging describes a chronic, systemic inflammatory state that results from agerelated changes of the immune system. It is thought to be the basis of various chronic inflammatory diseases of old age, e.g., atherosclerosis, diabetes mellitus type 2, as well as Alzheimer's disease and cancer. The development of this proinflammatory milieu and its progression is accelerated and in some cases aggravated by oxidative stress. Complex phytotherapeutics (such as Padma 28) act as pleiotropic compounds, suited to stop these pathogenic processes at different sites of action. Besides the clinical effect of Padma 28, different mechanisms of action of the preparation in atherosclerosis are well documented and research results support the hypothesis that a multi-target approach with herbal multicompounds may offer a valuable contribution to new prevention and treatment strategies in chronic inflammatory conditions. Herbal multicompounds are therefore well suited to positively influence "inflammaging" conditions and to slow down the development of ensuing diseases, in some cases also preventing them.

Keywords: Inflammaging, immunosenescence, chronic inflammation, geriatric diseases, Padma 28

hung stehen, haben zur Hypothese des "common soil” geführt. Diese besagt, dass Diabetes mellitus Typ 2, Arterio- sklerose und metabolisches Syndrom gemeinsame Ursachen haben, d.h. dass sie sich gewissermassen auf demselben 


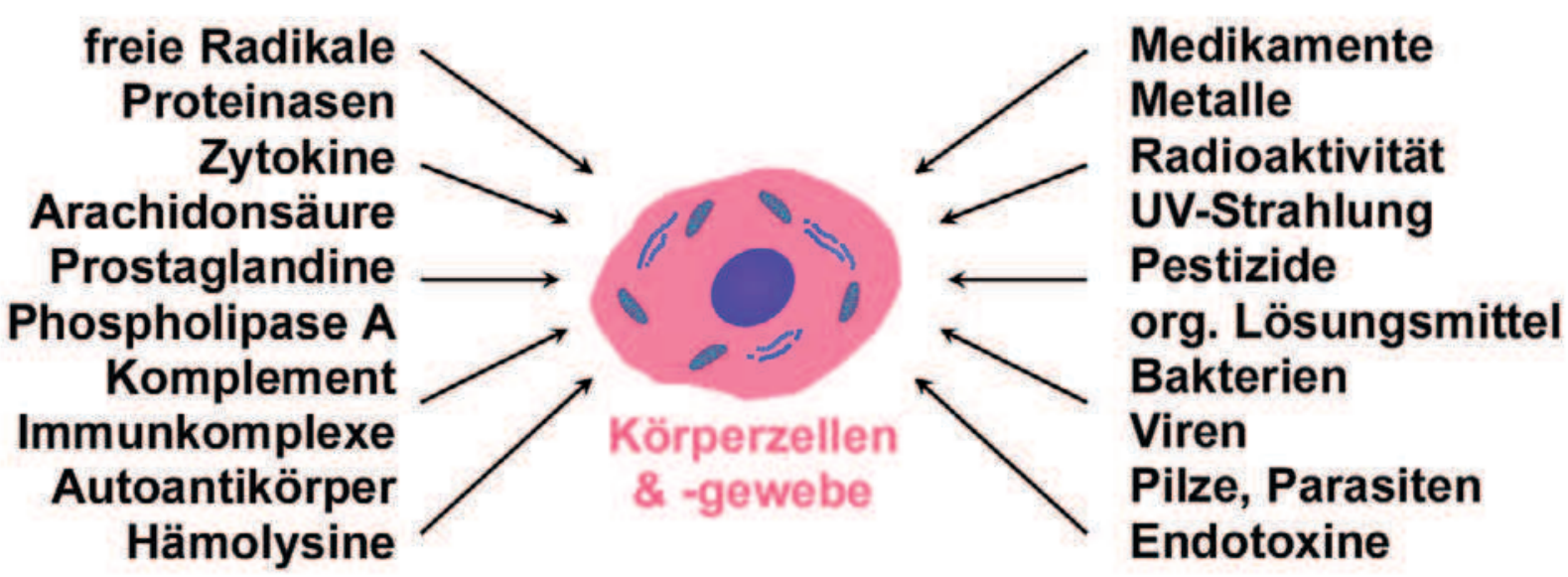

Abb. 1. Körperzellen und Gewebe sind konstant einer Vielzahl von endogenen und exogenen proinflammatorischen Noxen ausgesetzt, modifiziert nach $[17,18]$.

„Boden“ entwickeln [12,13]. Da auch bei ihnen Entzündungsprozesse pathogenetisch eine Rolle spielen, können im erweiterten Sinn noch andere Krankheiten der zweiten Lebenshälfte dazu gezählt werden, darunter Morbus Alzheimer, altersbedingte Makuladegeneration und Bluthochdruck wie auch verschiedene Tumorerkrankungen [1416]. Der entzündliche Basiszustand von Inflammaging könnte auch diesen chronisch-entzündlichen, im Alter zunehmenden Krankheiten als gemeinsame Ursache zu Grunde liegen, indem er die Anfälligkeit auf zusätzliche Entzündungsauslöser erhöht.

\section{Zell-/Gewebeschädigung bei Entzündungen: \\ Das synergistische Konzept}

Die Zellen und Gewebe des Körpers stehen temporär unter dem Einfluss von verschiedenen endogenen und exogenen Noxen. Freie Radikale, freigesetzte Zytokine, Faktoren des Komplementsystems oder Spaltprodukte des Arachidonsäurestoffwechsels führen zu Störeinflüssen an Biomembranen und im zellulären Signaltransfer. Exogene Noxen wie elektromagnetische oder radioaktive Strahlung, Industriegifte aber auch Mikroorganismen können zur Entstehung von freien Radikalen, zytotoxischen Stoffwechselprodukten und membranschädigenden Substanzen führen. Auch Autoimmunprozesse, Immunkomplexe und die Akti- vierung von autoimmunen zytotoxischen T-Zellen führen zu einer anhaltenden Aktivierung des Immunsystems und damit zu Zell- und Gewebeschädigung [17,18] (Abb. 1).

Bei der Beobachtung dieses „feindlichen Umfelds“, dem die Körperzellen ausgesetzt sind, insbesondere bei der Betrachtung der zytotoxischen Strategien von Immunzellen und Bakterien, fällt auf, dass beide ähnliche Mechanismen anwenden. Die Stoffe, die von Immunzellen zur Eliminierung von Entzündungstriggern freigesetzt werden sind teilweise dieselben, die pathogene Bakterien produzieren. Dies lässt vermuten, dass sowohl Immunzellen wie auch Mikroorganismen einen Synergismus zwischen verschiedenen Agenzien nutzen und eine Art proinflammatorischen Cocktail ausschütten, der u.a. Stoffe wie Oxidanzien, Proteinasen, kationische Peptide und Phospholipasen beinhaltet [17-20]. Die verschiedenen Komponenten dieses entzündlichen Stoffgemisches verstärken dabei ihre zytotoxische Wirkung gegenseitig (Abb. 2). Die Untersuchung dieses proinflammatorischen synergistischen Konzeptes sowie der Interaktionen zwischen Mikroorganismen und Immunzellen kann auch Erkenntnisse über die Mechanismen chronischer Entzündungsvorgänge sowie den Prozess des Inflammaging hervorbringen.

Ein Weg, auf dem sowohl Bakterien wie auch Immunzellen zytotoxisch wirken, führt über die Umwandlung von Plasminogen zu Plasmin, die über bak- terielle Kinasen bzw. über gewebespezifische Plasminogenaktivatoren und Proteinasen aktiviert wird. Plasmin wiederum aktiviert einerseits das Komplementsystem, andererseits kann es zusammen mit Oxidanzien die Wirkung zellschädigender Substanzen verstärken und Kollagenasen aktivieren [19]. Mit Hilfe solcher proteolytischen Enzyme können sich die Zellen frei durch das Gewebe bewegen und allenfalls eine Tumorstreuung begünstigen.

Neben Oxidanzien sind auch weitere Stoffgruppen wie etwa kationische Peptide am proinflammatorischen Cocktail von Immunzellen und Bakterien beteiligt. Kationische Peptide, z.B. Defensine, Elastase, Cathepsin G und sekretorische Phospholipase A2, können Bakterien, aber auch Körperzellen, angreifen und durch einen Synergismus mit Wasserstoffperoxid die zytotoxische Wirkung verstärken [17,21]. Kationische Peptide können ausserdem Bakterien opsonisieren, die wiederum die Phagozytose und den oxidativen Burst von Neutrophilen stimulieren.

\section{Entzündungszustand: Oxidativer Stress als Marker}

Oxidanzien wie etwa reaktive Sauerstoff- und Stickstoffspezies (ROS und RNS) oder Wasserstoffperoxid spielen eine wichtige Rolle bei der Zellschädigung durch Immunzellen oder Mikroorganismen. Einerseits dienen sie der Beseitigung von Immuntriggern, haben 


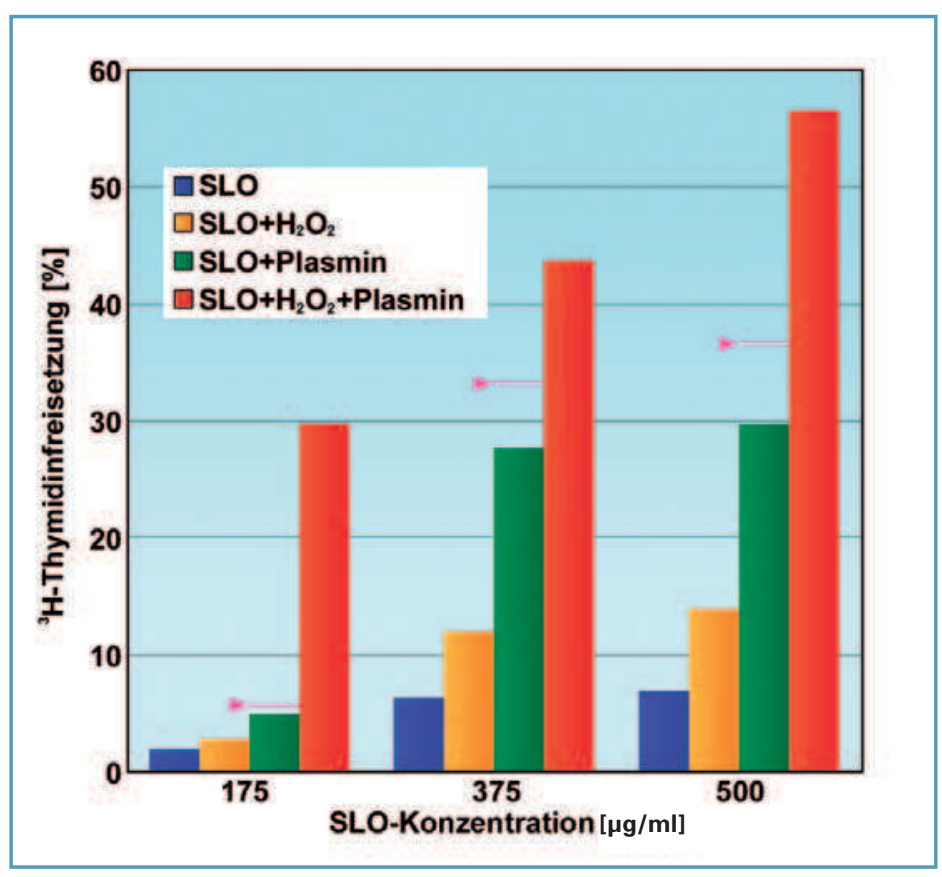

Abb. 2. Verschiedene zytotoxische Substanzen verstärken sich durch Synergismus gegenseitig in ihrer lytischen Wirkung. In Kombination (rote Balken) ist die Wirkung grösser als der bei additiver Wirkung erwartete Wert (rosa Pfeile). 3H-Thymidinfreisetzung wird als Mass für die Zellschädigung gewertet. (SLO: Streptolysin O).

zytotoxische Eigenschaften und halten die Entzündungskaskade am Laufen, andererseits begünstigen sie aber auch den Prozess der Immunoseneszenz und den proinflammatorischen Zustand des Inflammaging.

Zur Messung von oxidativem Stress bzw. der antioxidativen Kapazität von Lebensmitteln und arzneilichen Wirkstoffen sind viele Methoden bekannt [22]. Während der antioxidative Effekt z.B. eines Pflanzenextrakts durchaus Hinweise auf seine Wirkungsweisen geben kann, sagt eine solche Analyse wenig über den effektiven Einfluss auf die antioxidative Kapazität im Körper aus. Um Hinweise über den oxidativen Status im Körper zu erhalten, kann z.B. die Induktion redoxrelevanter Gene wie etwa Hämoxygenase-1 und Glutation herangezogen werden. Ein anderer Weg führt über die Ex-vivo-Messung der totalen antioxidativen Kapazität (TAC) von Körperflüssigkeiten und Zellen, wie sie mit verschiedenen Assays möglich ist. Hierbei kann der Status der natürlichen antioxidativen Kapazität von Körperflüssigkeiten wie Plasma oder Saliva abgeschätzt werden bzw. die Veränderung derselben nach Verabreichung von verschiede- nen Testsubstanzen. Im Experiment zeigt sich z.B. eine Erhöhung der TAC von Speichel nach der Einnahme von 4 Tabletten Padma 28 (1612 mg aktive Substanzen bzw. $2 / 3$ der maximalen Tagesdosis), wobei der stärkste Effekt drei Stunden nach der Einnahme festgestellt wurde [23] (Abb. 3).

Dieses Experiment wurde mit einer von unserer Gruppe an der Hebrew University Jerusalem entwickelten Methode durchgeführt. Im Gegensatz zu den meisten Assays zur Bestimmung der antioxidativen Kapazität von Stoffen, Pflanzen oder Körperflüssigkeiten, die auf der Messung von jeweils einer Art Oxidanzien, z.B. Peroxidverbindungen oder Hydroxylradikalen beruhen, wird hier ein oxidativer Cocktail eingesetzt, der simultan eine Vielzahl von Oxidanzien generiert. Mittels Chemiluminiszenz wird somit eine Reihe unterschiedlicher ROS und RNS gleichzeitig gemessen [23].

In diesem Chemiluminiszenz-basierten Test steigt das Signal unter Zugabe des oxidativen Cocktails rasant an und bleibt dann längere Zeit auf einem hohen Niveau bestehen. Die gleichzeitige Zugabe unterschiedlicher Antioxidanzien zeigte, dass diese den Anstieg des
Luminiszenzsignals dosisabhängig zeitlich verzögern und/oder die Erreichung des Maximalniveaus des Cocktails alleine verhindern. Das Ausmass der Verzögerung und Hemmung wurde als Mass für die antioxidative Kapazität der verschiedenen Testsubstanzen gewertet.

Gemessen wurden verschiedene, z.B. niedermolekulare Antioxidanzien, welche oft als Nahrungsergänzung gegeben werden, darunter Ascorbat (Vitamin C), Trolox (Vitamin E) und Glutathion. Ausserdem wurden weitere antioxidative Stoffe wie Harnsäure, und Extrakte aus verschiedenen Pflanzenpräparationen, wie etwa Wein und Tee, sowie pflanzliche Vielstoffgemische der Tibetischen Medizin, z.B. Padma 28 (Padma AG, Schwerzenbach) analysiert [24]. Dabei zeigte sich, dass multivalente Antioxidanzien aus pflanzlichen Extrakten im Allgemeinen eine höhere antioxidative Kapazität aufwiesen als monovalente niedermolekulare, bei welchen der Anstieg der Luminiszenzkurve zwar verzögert war, dann aber eine ähnliche Form hatte wie beim proinflammatorischen Cocktail alleine (Abb. 4). Dies deutet darauf hin, dass antioxidative Monosubstanzen wie z.B. Vitamin E, Glutathion und Harnsäure nach einer gewissen Zeit aufgebraucht sind und keine Regenerationsfähigkeit in sich tragen.

Bei komplex zusammengesetzten Antioxidanzien, wie etwa bei Padma 28, erfolgte der Anstieg der Luminiszenz nicht nur verzögert, sondern auch flacher, so dass die Kurve den Level des proinflammatorischen Cocktails nicht erreichte. Hier scheint die TAC nicht durch die vom Cocktail generierten Oxidanzien aufgebraucht zu werden, vermutlich auf Grund der Zusammensetzung aus Antioxidanzien unterschiedlicher Stärke, die sich gegenseitig kaskadenförmig stützen und regenerieren [25]. Interessanterweise waren auch hier die Antioxidanzien viel effektiver, wenn sie gleichzeitig in Kombination eingesetzt wurden, was wiederum die Bedeutung von synergistischen Konzepten beim Schutz vor oxidativem Stress und damit bei chronischen Entzündungsprozessen betont $[17,26]$.

Die komplexe Zusammensetzung 


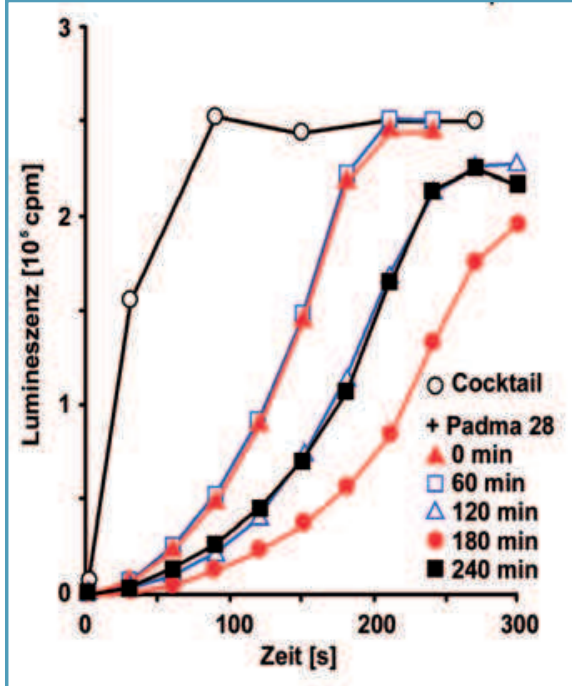

Abb. 3. Die Einnahme von 4 Tabletten Padma 28 (1612 mg aktive Substanzen) erhöht die totale antioxidative Kapazität von Speichel mit einem maximalen Effekt drei Stunden nach der Einnahme.

führt nicht nur dazu, dass pflanzliche Vielstoffgemische unter oxidativem Stress robuster reagieren, sie scheint auch für die die Vielfalt an Wirkmechanismen vieler Pflanzenpräparate verantwortlich zu sein. Diese Pleiotropie, d.h. ein Präparat hat viele verschiedene Wirkorte zugleich, konnte am Beispiel der antiatherogenen Wirkungsmechanismen von Padma 28 gezeigt werden [28]. Phytotherapeutika, im Besonderen Vielpflanzengemische, wie sie in asiatischen Medizinsystemen verwendet werden, beeinflussen komplexe Vorgänge wie etwa diejenigen in chronischen Entzündungszuständen und Inflammaging an verschiedenen Orten im Körper zugleich und wirken über unterschiedliche Mechanismen. Deshalb scheinen sie besonders geeignet, um synergistische pathogenetische Prozesse zu unterbrechen. Da pleiotrope Stoffe mehrere Zielorte aufweisen, werden sie auch als „multi-target drugs“ bezeichnet

\section{Multi-target-Strategien gegen zytotoxische entzündliche Synergismen}

Im Gegensatz zu multi-target-Präparaten werden single-target-Therapien den synergistischen proinflammatorischen Mechanismen bei komplexen Erkran-

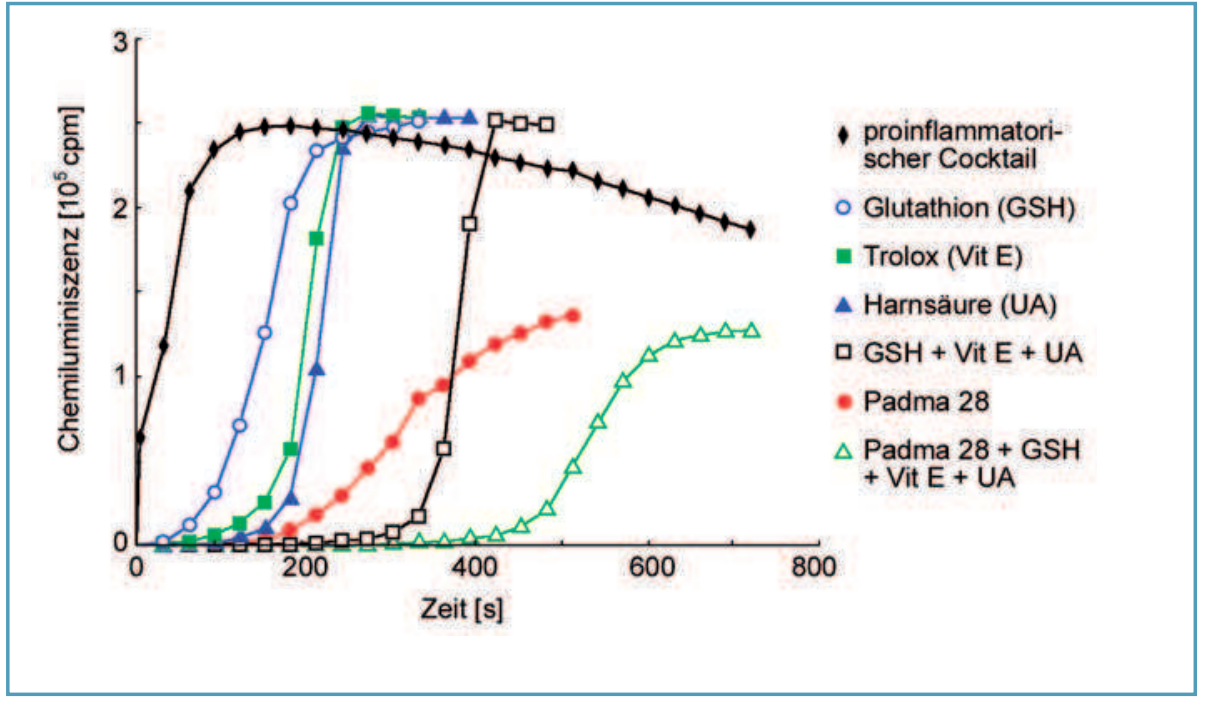

Abb. 4. Die Generierung von Oxidanzien durch einen standardisierten oxidativen Cocktail (detaillierte Methode siehe [23]) wird durch die Zugabe von Antioxidanzien verzögert. Während die Einzelsubstanzen Glutathion, Trolox und Harnsäure mit der Zeit verbraucht werden, hat die Pflanzenformel Padma 28 eine längere Wirkung. Besonders effektiv sind die analysierten Substanzen, wenn sie in Kombination eingesetzt werden.

kungen nicht gerecht $[27,29]$. Da sie nicht nur die proinflammatorischen Regelkreise simultan an verschiedenen Stellen unterbrechen können, sondern im Allgemeinen auch eine gute Verträglichkeit aufweisen, kommt pflanzlichen Präparaten bei solchen vielschichtigen pathogenetischen Prozessen eine besondere Bedeutung zu. Insbesondere Pflanzenformeln der Tibetischen Medizin, die den Synergismus zwischen ihren verschiedenen Komponenten ausnutzen und dabei die Nebenwirkungen einzelner Komponenten mindern, können hier neue Behandlungsansätze darstellen. Das multi-target-Konzept wird auch in der modernen westlichen Pharmakotherapie immer wichtiger. Obwohl diese lange Zeit dem Schlüssel-Schloss-Prinzip verhaftet war, das chemisch genau definierte Substanzen mit grossem Effekt an einem Ziel anwendet, setzt sie im Licht der zunehmenden multifaktoriellen Erkrankungen mehr und mehr auf multi-target-Strategien. So werden z.B. bei Virushepatitis C oder HIV standardmässig Kombinationstherapien angewendet [30,31].

Im weiteren Sinn können auch Nahrungsmittel und Getränke pflanzlichen Ursprungs als multi-target-Präparate aufgefasst werden und die Diät als Medizin begriffen werden. Eine Auffassung, die in der Erfahrungsmedizin alt- bekannt ist und dazu führt, dass neben Kräuterformeln die Beratung bezüglich Nahrung und Verhalten einen wichtigen Bestandteil der Behandlung bilden. Die Bedeutung der Ernährung nimmt auch in der westlichen Medizin wieder zu und ist vermehrt Gegenstand der Forschung. Unter dem Begriff "French paradox" ist z.B. das Phänomen bekannt, dass in Frankreich trotz hohem Anteil an gesättigten Fettsäuren in der Nahrung und hohem Alkoholkonsum eine geringere Inzidenz von Herz-Kreislauferkrankungen festgestellt wurde, als im Rest von Mitteleuropa [32]. Als Ursache dafür wird ein regelmässiger Konsum von Rotwein angenommen. Verschiedene Mechanismen scheinen zum protektiven Effekt von Rotwein bei Herz-Kreislauferkrankungen beizutragen. Neben der gefässerweiternden Wirkung des Alkohols haben Rotweinpolyphenole wie z.B. Resveratrol und Proanthocyanidine einen positiven Einfluss auf die Endothelfunktion, reduzieren die Thrombozytenaggregation und können durch eine antioxidative Wirkung Biomoleküle vor Veränderungen durch Radikalstress schützen. Bezüglich Arteriosklerose wird dabei der Verminderung der oxidativen Modifizierung von LDL-Cholesterin eine besondere Bedeutung zugemessen [33,34].

Die im Allgemeinen kleine Absorp- 
tionsrate von Polyphenolen spricht gegen eine solche direkte Wirkung. Pflanzliche Antioxidanzien wirken jedoch nebst direkten Effekten auch auf indirekte Weise im Körper. So wurde gezeigt, dass Weinpolyphenole im Magen die Aufnahme von Hydroperoxiden und Aldehyden hemmen und so die LDL-Oxidation vermindern [35]. Ein weiteres Beispiel für die indirekte Wirkung von Pflanzen konnte für den Konsum von Äpfeln gezeigt werden, der eine Erhöhung der Konzentration von körpereigenen Antioxidanzien im Plasma bewirkte [36].

Wie schon erwähnt, sind Oxidanzien nur eine von vielen Komponenten des synergistischen proinflammatorischen Cocktails im Entzündungsgeschehen. Komplexe Pflanzenformeln haben in der Regel nicht nur antioxidative Eigenschaften, sondern können verschiedene Mechanismen im synergistischen Entzündungsgeschehen gleichzeitig beeinflussen. Dank der über 30-jährigen Forschungstätigkeit mit dem Produkt kann das Vielstoffpräparat Padma 28, eine Formel aus der Tibetischen Medizin, als Beispiel für eine derartige synergistische Wirkweise dienen (Abb. 5).

Die extensive Datenlage zu Padma 28 stützt die Hypothese, dass pflanzliche multi-target-Präparate besonders bei multifaktoriellen Störungen, wie chronische Entzündungen, eine valide Behandlungsoption darstellen. Durch die multi-target-Wirkung können sie auch präventiv eingesetzt werden, besonders im Rahmen der Verlangsamung und Vorbeugung von Inflammaging sowie seiner Folgeerkrankungen.

\section{Prävention und Therapie von Inflammaging und chronischen Erkrankungen}

Weil Inflammaging als Basis für eine Vielzahl von so genannten Alterserkrankungen vermutet wird, kann die Verminderung dieses chronischen Entzündungszustandes im Alter bzw. die Verlangsamung seiner Entstehung viel zur Prävention von Erkrankungen wie z.B. Arteriosklerose, Morbus Alzheimer und Diabetes mellitus 2 beitragen.

Verschiedene Daten unterstützen die Hypothese eines "multi-target"-An-

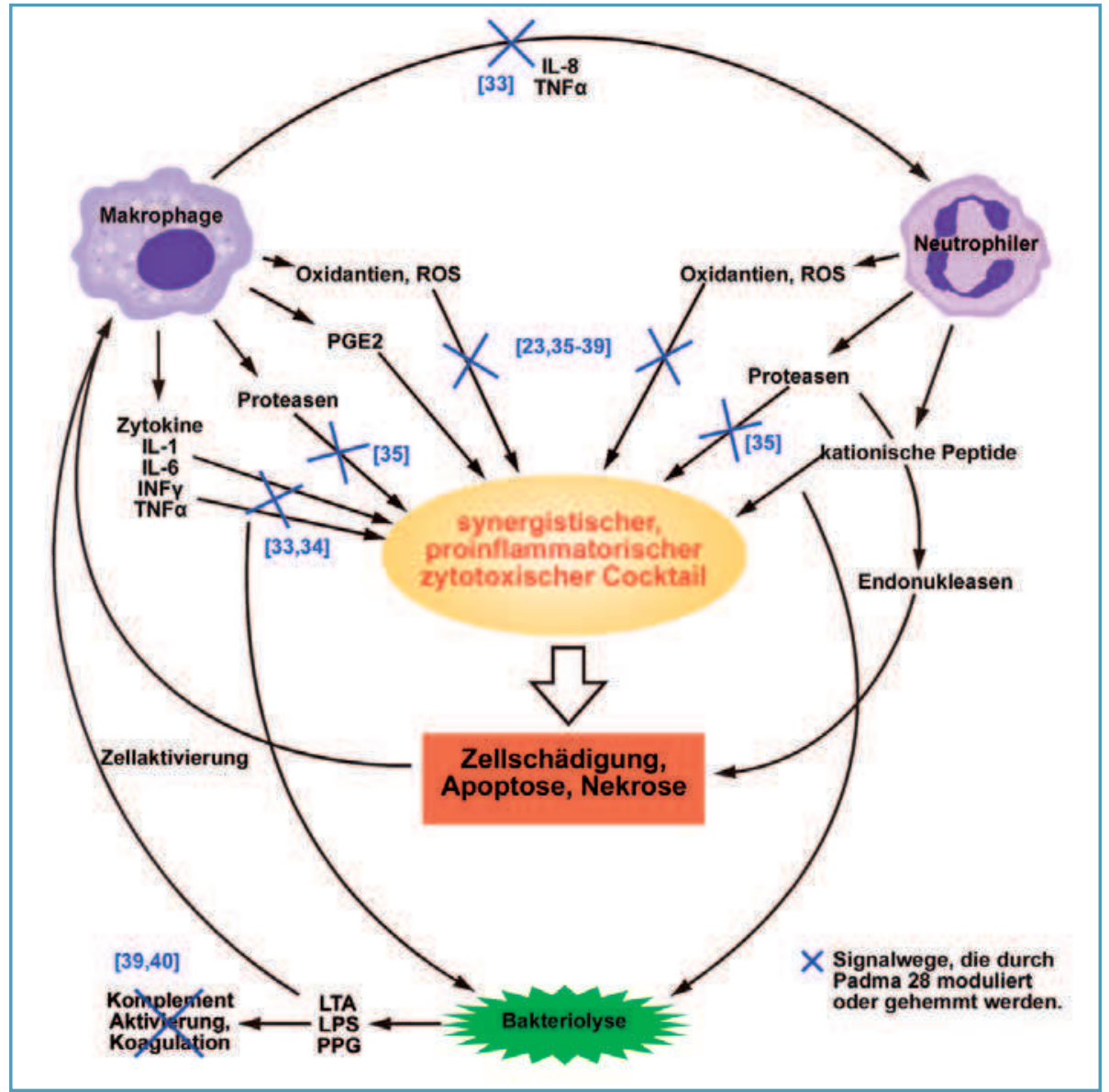

Abb. 5. Feedback-Mechanismen in chronischen Entzündungsprozessen. Makrophagen und neutrophile Granulozyten setzen einen entzündlichen Cocktail frei, an dem auch Bakterien beteiligt sein können. Die antientzündliche Wirkung des multi-target-Präparats Padma 28 wird durch die blauen Kreuze markiert [23,37-44]. (ROS: reaktive Sauerstoffspezies; PGE2: Prostaglandin 2; IL: Interleukin; LTA: Lipoteichonsäure, LPS: Lipopolysaccharid; PG: Peptidoglycan).

satzes mit pflanzlichen Vielstoffgemischen bei chronisch-entzündlichen Prozessen. Insbesondere weisen sie darauf hin, dass komplexe Pflanzenpräparate hier besser geeignet sind als z.B. mono-valente Antioxidanzien. So zeigten etwa klinische Studien mit antioxidativen monomolekularen Substanzen, wie Vitamin C oder E, enttäuschende Resultate bezüglich Inzidenz chronischer Erkrankungen und Gesamtmortalität [45-47]. Weitere Hinweise auf die Bedeutung pflanzlicher multi-target-Präparate geben die Erkenntnisse aus epidemiologischen Untersuchungen und Studien mit pflanzlichen Nahrungsmitteln [32,34-36].

Um den Prozess der Immunoseneszenz zu verlangsamen und Inflammaging zu hemmen, muss oxidativer Stress und die Persistenz von Immuntriggern vermieden werden. Neben Veränderungen im Alltag stellen Phyto- kombinationen wertvolle Faktoren in solchen anti-inflammatorischen Behandlungs- und Präventionsansätzen dar. Da sie die synergistischen inflammatorischen Abläufe bei Inflammaging wie auch bei chronisch entzündlichen Erkrankungen in einer multi-targetWeise an verschiedenen Stellen durchbrechen, können pleiotrope Pflanzenformeln, vorzugsweise solche mit geprüfter Qualität und Sicherheit, den Formenkreis von Inflammaging günstig beeinflussen und eine wichtige Rolle bei der Vermeidung und der Behandlung von chronisch-entzündlichen Alterserkrankungen spielen.

\section{Literatur}

1 Candore G, Colonna-Romano G, Balistreri CR, Di Carlo D, Grimaldi MP, Listì F, Nuzzo D, Vasto S, Lio D, Caruso C. Biology of longevity: role of the innate immune system. Rejuvenation Res 2006:9:143-8. 
2 Franceschi C, Valensin S, Fagnoni F, Barbi C, Bonafè M. Biomarkers of immunosenescence within an evolutionary perspective: the challenge of heterogeneity and the role of antigenic load. Exp Gerontol 1999;34:911-21.

3 Capri M, Monti D, Salvioli S, Lescai F, Pierini M, Altilia S, Sevini F, Valensin S, Ostan R, Bucci L, Franceschi C. Complexity of antiimmunosenescence strategies in humans. Artif Organs 2006;30:730-42.

4 De Martinis M, Franceschi C, Monti D, Ginaldi $\mathrm{L}$. Inflamm-ageing and lifelong antigenic load as major determinants of ageing rate and longevity. FEBS Lett 2005;579:2035-9.

5 Giunta S. Is inflammaging an auto[innate]immunity subclinical syndrome? Immun Ageing 2006:3:1-2

6 Johansen JS, Pedersen AN, Schroll M, Jørgensen T, Pedersen BK, Bruunsgaard $\mathrm{H}$. High serum YKL-40 level in a cohort of octogenarians is associated with increased risk of allcause mortality. Clin Exp Immunol 2008;151: 260-6.

7 Dinarello CA. Interleukin 1 and interleukin 18 as mediators of inflammation and the aging process. Am J Clin Nutr. 2006;83:447S-455S.

8 Franceschi C, Bonafè M, Valensin S. Human immunosenescence: the prevailing of innate immunity, the failing of clonotypic immunity, and the filling of immunological space. Vaccine 2000;18:1717-20.

9 Pedersen BK. The anti-inflammatory effect of exercise: its role in diabetes and cardiovascular disease control. Essays Biochem 2006;42: 105-17.

10 Petersen AM, Pedersen BK. The role of IL-6 in mediating the anti-inflammatory effects of exercise. J Physiol Pharmacol 2006;57 Suppl 10:43-51.

11 Simpson N, Dinges DF. Sleep and inflammation. Nutr Rev 2007;65:S244-52.

12 Stern MP. Diabetes and cardiovascular disease. The "common soil" hypothesis. Diabetes 1995;44:369-74

13 Ceriello A, Motz E. Is oxidative stress the pathogenic mechanism underlying insulin resistance, diabetes, and cardiovascular disease? The common soil hypothesis revisited. Arterioscler Thromb Vasc Biol 2004;24:816-23.

14 Shoenfeld Y, Sherer Y, Harats D. Artherosclerosis as an infectious, inflammatory and autoimmune disease. Trends Immunol 2001;22: 293-5.

15 Das UN. Acetylcholinesterase and butyrylcholinesterase as possible markers of lowgrade systemic inflammation. Med Sci Monit. 2007;13:RA214-21.

16 Boccardo E, Villa LL. Viral origins of human cancer. Curr Med Chem 2007;14:2526-39.

17 Ginsburg I, Kohen R. Cell damage in inflammatory and infectious sites might involve a coordinated "cross-talk" among oxidants, microbial haemolysins and ampiphiles, cationic proteins, phospholipases, fatty acids, proteinases and cytokines (an overview). Free Radic Res 1995;22:489-517.

18 Ginsburg I. Could synergistic interactions among reactive oxygen species, proteinases, membrane-perforating enzymes, hydrolases, microbial hemolysins and cytokines be the main cause of tissue damage in infectious and inflammatory conditions? Med Hypotheses 1998;51:337-46.

19 Ginsburg I, Misgav R, Pinson A, Varani J, Ward PA, Kohen R. Synergism among oxidants, proteinases, phospholipases, microbial hemolysins, cationic proteins, and cytokines. Inflammation 1992;16:519-38.

20 van der Poll T, Opal SM. Host-pathogen inter- actions in sepsis. Lancet Infect Dis 2008;8: $32-43$

21 Hancock RE. Cationic peptides: effectors in innate immunity and novel antimicrobials. Lancet Infect Dis 2001;1:156-64.

22 Huang D, Ou B, Prior RL. The chemistry behind antioxidant capacity assays. J Agric Food Chem 2005;53:1841-56.

23 Ginsburg I, Sadovnic M, Oron M, Kohen R. Novel chemiluminescence-inducing cocktails, part II: measurement of the anti-oxidant capacity of vitamins, thiols, body fluids, alcoholic beverages and edible oils. Inflammopharmacology 2004;12:305-20.

24 Koren E, Zverev I, Ginsburg I, Kohen R. Supplementation with antioxidants fails to increase the total antioxidant capacity of several cell lines in culture. Biomed Pharmacother 2008;62:179-88.

25 Vennos C, Schwabl H. Chronische Low-Level Entzündungen: Pflanzliche Vielstoffgemische als integrative Erweiterung antioxidativer Strategien. Schweiz Zschr GanzheitsMedizin 2006:18:333-8.

26 Schwabl H, Vennos C. Der "multi-target"Ansatz tibetischer Heilmittel: Wirkmechanismen von Padma 28 im entzündlichen Geschehen am Beispiel der Arteriosklerose. Schweiz Zschr GanzheitsMedizin. 2006;18: 213-8

27 Liu M, Slutsky AS. Anti-inflammatory therapies: application of molecular biology techniques in intensive care medicine. Intensive Care Med 1997;23:718-31.

28 Ueberall F, Fuchs D, Vennos C. Das anti-inflammatorische Potential von Padma 28 Übersicht experimenteller Daten zur antiatherogenen Wirkung und Diskussion des Vielstoffkonzepts. Forsch Komplementärmed 2006;13(Suppl 1):7-12.

29 Ginsburg I. Multi-drug strategies are neces sary to inhibit the synergistic mechanism causing tissue damage and organ failure in post infectious sequelae. Inflammopharmacology 1999;7:207-17.

30 Waters L, Nelson M. New therapeutic options for hepatitis C. Curr Opin Infect Dis. 2006; 19:615-22.

31 De Clercq E. Anti-HIV drugs. Verh K Acad Geneeskd Belg. 2007;69:81-104.

32 Richard JL. Coronary risk factors. The French paradox. Arch Mal Coeur Vaiss 1987;80: 17-21.

33 Gazzieri D, Trevisani M, Tarantini F, Bechi $P$ Masotti G, Gensini GF, Castellani S, Marchionni N, Geppetti P, Harrison S. Ethanol dilates coronary arteries and increases coronary flow via transient receptor potential vanilloid 1 and calcitonin gene-related peptide. Cardiovasc Res. 2006 Jun 1;70:589-99.

34 Vidavalur $\mathrm{R}$, Otani $\mathrm{H}$, Singal PK, Maulik N. Significance of wine and resveratrol in cardiovascular disease: French paradox revisited. Exp Clin Cardiol 2006;11:217-225.

35 Gorelik S, Ligumsky M, Kohen R, Kanner J. A novel function of red wine polyphenols in humans: prevention of absorption of cytotoxic lipid peroxidation products. FASEB J 2008;22: 41-6.

36 Lotito SB, Frei B. Consumption of flavonoidrich foods and increased plasma antioxidant capacity in humans: cause, consequence, or epiphenomenon? Free Radic Biol Med. 2006 41:1727-46

37 Barak V, Kalickman I, Halperin T, Birkenfeld S, Ginsburg I. PADMA-28, a Tibetan herbal preparation is an inhibitor of inflammatory cytokine production. Eur Cytokine Netw 2004;15: 203-9.

38 Neurauter G, Wirleitner B, Schroecksnadel K,
Schennach H, Ueberall F, Fuchs D. PADMA 28 modulates interferon-gamma-induced tryptophan degradation and neopterin production in human PBMC in vitro. Int Immunopharmacol 2004:4:833-9.

39 Ginsburg I, Sadovnik M, Sallon S, Milo-Goldzweig I, Mechoulam R, Breuer A, Gibbs D, Varani J, Roberts S, Cleator E, Singh N. PADMA-28, a traditional Tibetan herbal preparation inhibits the respiratory burst in human neutrophils, the killing of epithelial cells by mixtures of oxidants and pro-inflammatory agonists and peroxidation of lipids. Inflammopharmacology 1999;7:47-62.

40 Brunner-La Rocca HP, Schindler R, Schlumpf M, Saller R, Suter M. Effects of the Tibetan herbal preparation Padma 28 on blood lipids and lipid oxidisability in subjects with mild hypercholesterolaemia. VASA 2005;34:11-7.

41 Moeslinger T, Friedl R, Volf I, Brunner $M$ Koller E, Spieckermann PG. Inhibition of inducible nitric oxide synthesis by the herbal preparation Padma 28 in macrophage cell line. Can J Physiol Pharmacol 2000;78:861-6.

42 Suter M, Richter C. Anti- and pro-oxidative properties of PADMA 28, a Tibetan herbal formulation. Redox Rep 2000;5:17-22.

43 Winther $\mathrm{K}$, Kharazmi A Himmelstrup $\mathrm{H}$, Drabaek H, Mehlsen J. PADMA-28, A botanical compound, decreases the oxidative burst response of monocytes and improves fibrinolysis in patients with stable intermittent claudication. Fibrinolysis 1994;8(Suppl 2):47-9.

44 Altermatt $R$, von Felten A. In-Vitro-Untersuchungen mit Padma 28: Hemmung der Thrombozytenfunktion. Schweiz Zschr GanzMed 1992;7/8(Suppl 1):7-12.

45 Marchioli R, Schweiger C Levantesi G, Tavazzi $L$, Valagussa F. Antioxidant vitamins and prevention of cardiovascular disease: epidemiological and clinical trial data. Lipids 2001;36 Suppl:S53-63.

46 Miller ER 3rd, Pastor-Barriuso R, Dalal D, Riemersma RA, Appel LJ, Guallar E. Metaanalysis: high-dosage vitamin $\mathrm{E}$ supplementation may increase all-cause mortality. Ann Intern Med 2005;142:37-46.

47 Bleys J, Miller ER, Pastor-Barriuso R, Appel LJ, Guallar E. Vitamin-mineral supplementation and the progression of atherosclerosis: a meta-analysis of randomized controlled trials. Am J Clin Nutr 2006;84:880-7.

\section{Disclosure statement}

Cécile Vennos is an employee of Padma AG. The other authors declare that they have no financial or other conflict of interest in relation to the content of this article.

\section{Korrespondenzadresse:}

Dr. Cécile Vennos

Padma AG, Research Department

Wiesenstrasse 5, CH-8603 Schwerzenbach c.vennos@padma.ch 